\title{
Effectiveness Evaluation of Air and Missile Defense System Based on Parametric Diagrams
}

\author{
Lu Fa, a , Sun Wenhu ${ }^{1, b}$ \\ ${ }^{1}$ Academy of Logistics, Beijing 100858, China. \\ alufa08@qq.com, bigerswh@163.com
}

Keywords: parametric model, SysML, weapon system effectiveness evaluation.

\begin{abstract}
Under the complex operational environment of the antimissile operation, the application of Systems Modeling Language (SysML) parametric model in weapon system effectiveness evaluation is explored. The function of parametric graph model, the construction process of the model and the optimization method of the model are researched. Using feedback mechanism to collect, collate and calculate the parameters of the model, and realize the leap from the index relationship description to the calculation of the index. Finally, the feasibility and effectiveness of the proposed method is verified by using the system of antimissile weapon system as an example.
\end{abstract}

\section{Introduction}

With the rapid development of space technology and the continuous development of missile technology and under the high-tech war background ${ }^{[1]}$, using missiles to destroy critical operational facilities has become an important form of combat. In order to achieve victory in the war in the future, to prevent our own key objectives be destroyed causing a rapid decline in the combat effectiveness, we must intercept and destroy the enemy missiles in launch process. The core features of antimissile operations is scout and pre-alarming integration, command and control command integration and comprehensive fire application ${ }^{[2][3]}$. As a new type of cutting-edge technology application, it is necessary to make a scientific demonstration before the construction of air and missile defense weapon system. In American industry the definition of system effectiveness is: a function of the reliability and validity of the system and the degree of completing a set of tasks. The analysis and evaluation of the effectiveness of the air and missile defense system has important significance for the deployment, evaluation, development and optimization of anti missile weapon system.

\section{parametric diagram model}

SysML is a new system engineering standard modeling language proposed by the International Council on Systems Engineering (INCOSE) and The object management organization(OMG) based on the reuse and extension of UML2.0.

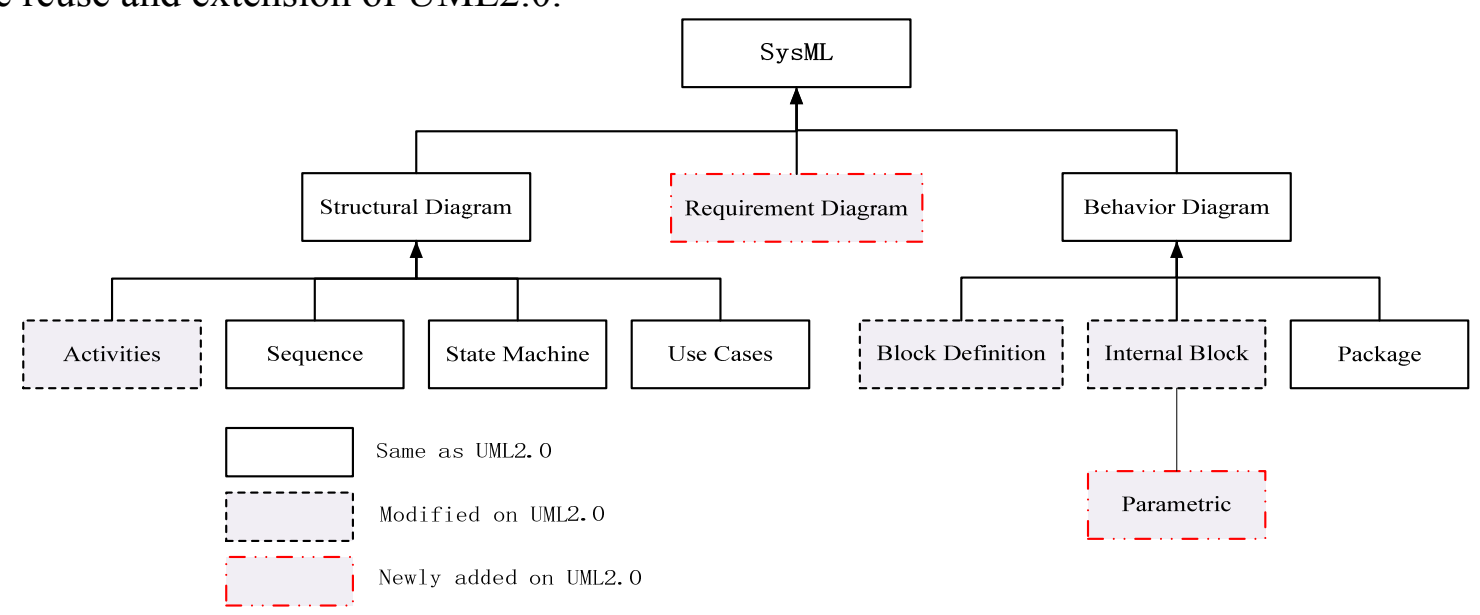

Fig.1 SysML structure view 
SysML defines nine types graphics to visualize a systems engineering model, as shown in Fig. 1. Parametric graph is a unique view model of SysML, which is based on the existence of object attributes and the relationship between the attributes of each object in the system. The parametric graph ordinary use to directing relationship between object attributes in order to analyze the function, measure the effectiveness and clarify the relationship among the variables.

\section{Construction of parametric diagram model}

Parametric diagram represents the relationship between the attributes of each object in the system, which determines that the argument graph is not an isolated existence, but rather the relationship between them. In IBM Rational Rhapsody modening tool the graphical elements used by the parameter diagram are called constraint block, and these models are defined by general or basic mathematical formulas. The purpose of parametric drawing is to analyze the key parameters of system, including performance evaluation, reliability evaluation and physical property evaluation. Evaluation is an indispensable part of the system engineering process, UML and IDEF modeling language has no parameter diagram, so they can't carry out engineering analysis. In the previous modeling language, there is no standardization of engineering analysis model, this leads to the failure to associate the system static structure model and the structure behavior of the system and leads to serious mismatch between structure and behavior. Furthermor, system structure model and engineering analysis model can't be unified and synchronized design, these increase the complexity and dissimilarity of the system, so the analysis model has to be constructed independently in the engineering analysis. This traditional approach lead to the increase in the number of engineering analysis models and easy to appear the structure model and the behavior model inconsistent phenomenon. In the process of system internal structure modeling in Rhapsody, parametric diagram model describes what attributes the system has and what is the relationship between these attributes. The constraint relations in parametric model are expressed by equation, the parameters in the equation are the system attributes and the equation itself describes the constraint relationship of the attributes.

When building parametric diagram, parameter attributes in constrained blocks are derived from the properties of the parameters in the block diagram. Attributes in the parameter model and the attributes in the block diagram are actually placed in different places of the same object, They are related to each other. The parameters of the other parts are changed as the parameters are modified. Such mechanisms ensure the consistency of the relationship between the parameters and the parameters value in the whole process of the system modeling. By establishing system parametric diagram model, it can clearly reflect the relationship between the properties of the system and the properties of the system parameters. When the performance parameters of the system are analyzed, the performance and efficiency of the system can be quantitatively analyzed.

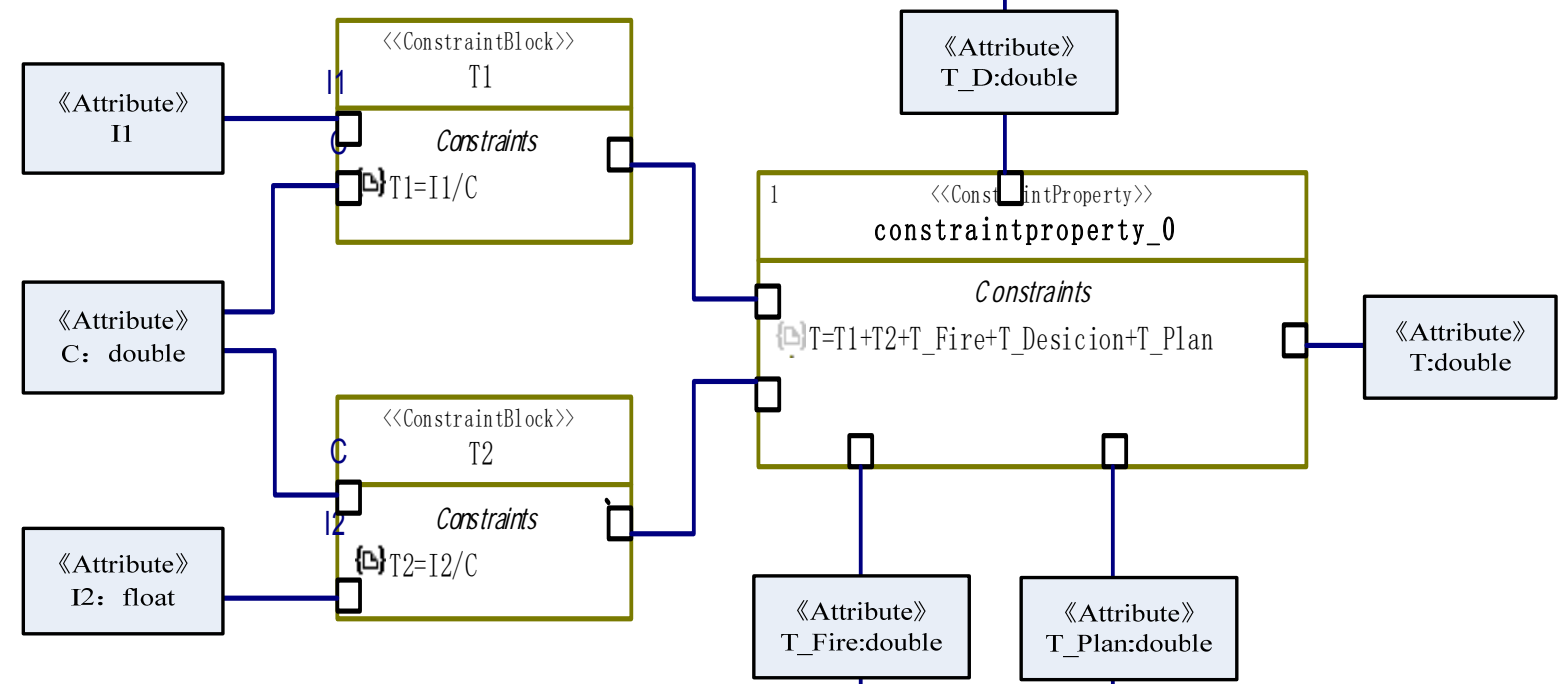

Fig.2 A simple parameter diagram 
Fig. 2 shows a simple parameter diagram.The boxes "Attributes" represent the attributes parameter of system, the boxes "ConstraintBlock" represent the constraints of parameters, which represents the constraint equations between attributes. In Fig.2, the constraint equation of the constraint equation is $\mathrm{T} 1=\mathrm{I} 1 / \mathrm{C}$, that is, the value of and $\mathrm{I} 1 \mathrm{can}$ be calculated by $\mathrm{T} 1$ and $\mathrm{C}$. According to the constraint equation can calculate the value of the target attribute or the optimal value of the target attribute according to the constraint relationship.

\section{Construction and analysis of effectiveness evaluation model of air and missile defense model based on parametric diagram}

In this paper, We use parametric diagram model to evaluate the effectiveness of high-level terminal interception phase and calculate the successful probability of intercepting an enemy incoming missile. As shown in Fig.3, the parameter block definition diagram describes the performance parameters of the key equipment in the antimissile equipment system, which includes the constraint relationship between the parameters of each module and the type and property of the system.

As shown in Fig.3, in the parameter block "WarningRadar", we calculate the probability of discovering targets (Pd). Use some performance parameters of early warning radar such as target echo power(Snr), noise power(S) and minimal signal-to-noise ratio reliability( Npr). Through the calculation of the corresponding constraint formula in the block, the probability of the early warning radar to discover the target is eventually obtained. The constraint formula is as form (1):

$$
P_{d}=\exp \left(-\left(\operatorname{Snr} /\left(1+\left(S / N_{p r}\right)\right)\right)\right)
$$

The "Energy Test Equipment" parameter block is defined under the parameter block "WarningRadar" to calculate noise power. The constraint formula is as follows:

$$
N_{p r}=B \times B_{n r} \times N_{p} \times R_{b}
$$

In the form (2), $\mathrm{Npr}$ express noise power, $\mathrm{B}$ express Boltzmann constant, $B=1.38 \times 10^{\wedge}(-23) \mathrm{J} / \mathrm{k}$ 。 $B_{n r}$ express reference noise figure, $N_{p}$ express noise figure, $R_{b}$ express receiver bandwidth.

The purpose of "Defence Missile" is calculate missile destroy probability $P_{k}$. L express missile killing radius, $G$ express guidance accuracy. The constraint formula is as follows:

$$
P_{k}=L / G
$$

Finally, we use "Air Defence System" parameter block to calculate the successful probability of intercepting an enemy incoming missile (Ps). The constraint formula is as follows:

$$
P_{s}=P_{d} / P_{k}
$$

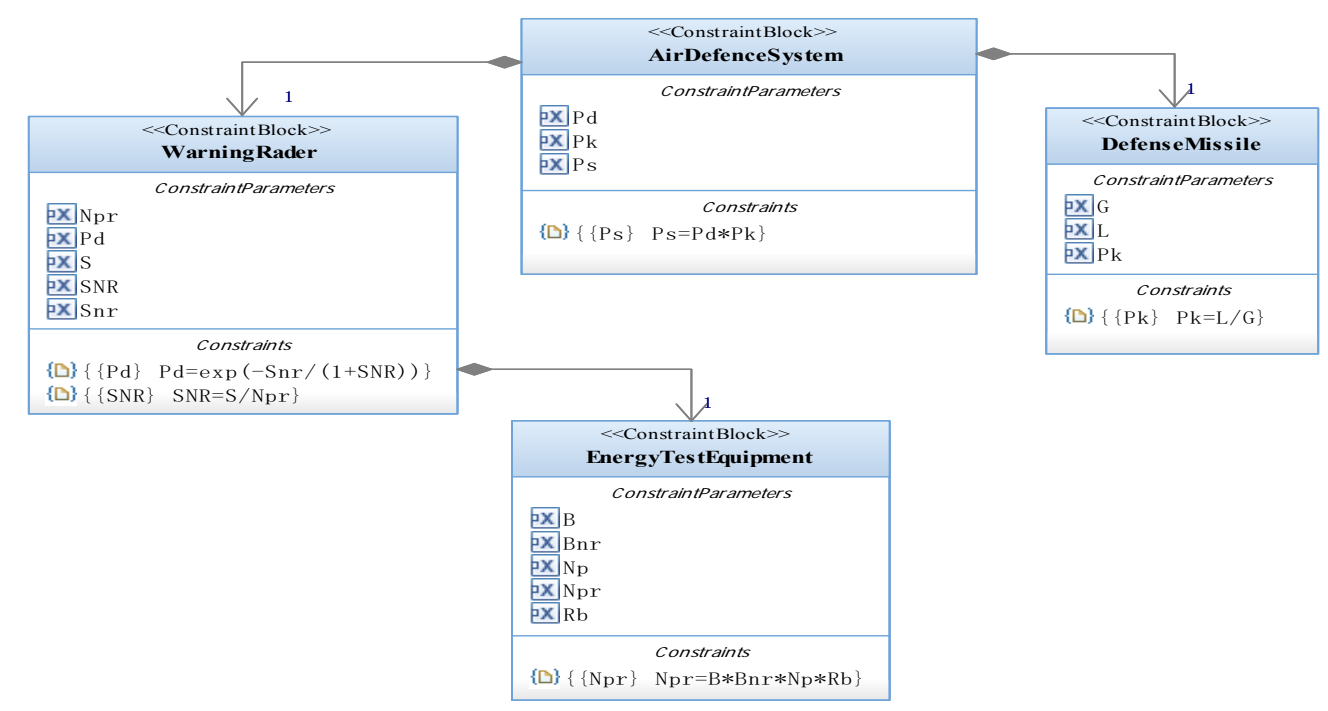

Fig.3 Parameter block definition diagram 
The next step is create parameterized diagram for constraint block. As shown in Fig.4.

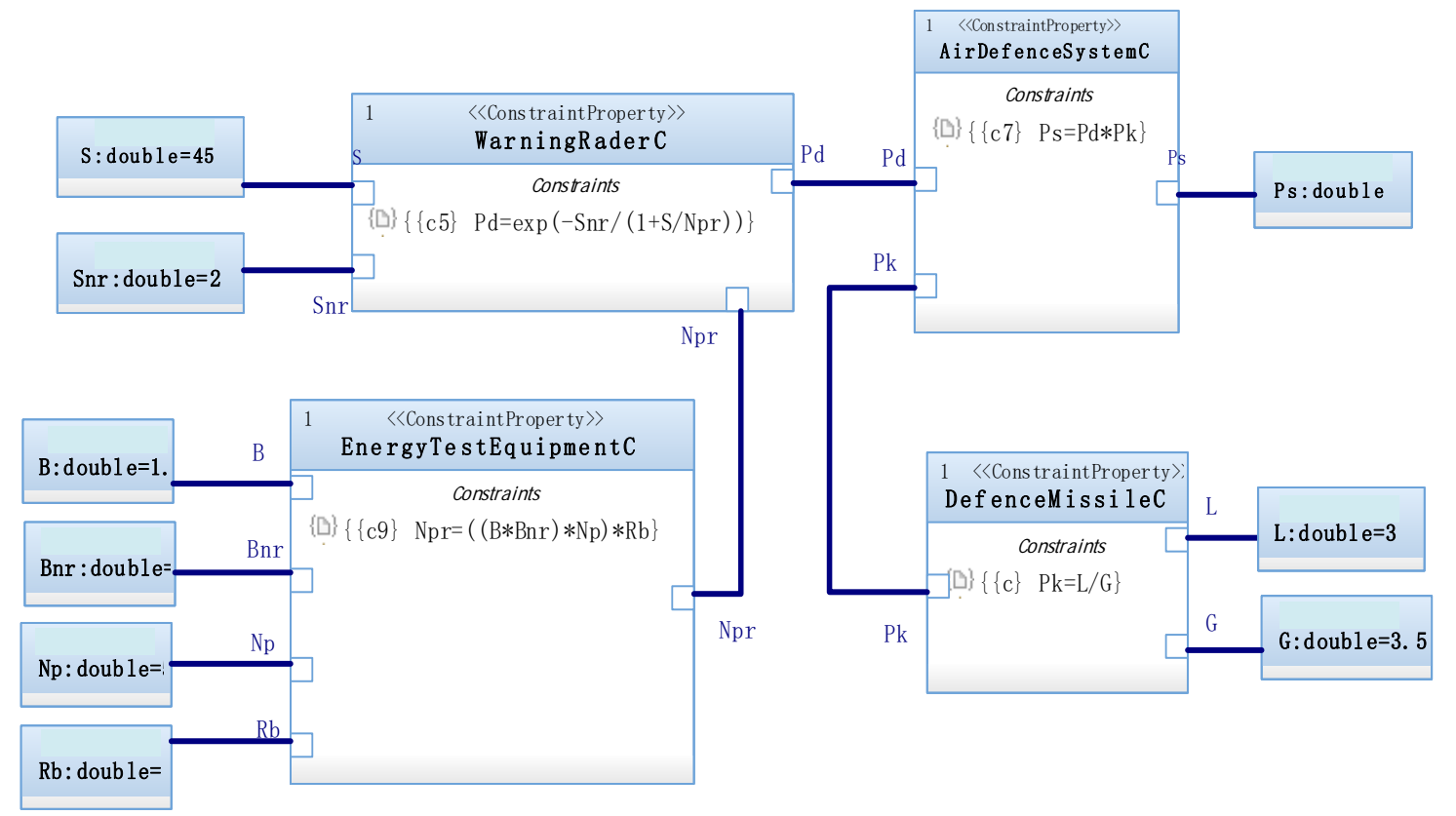

Fig.4 Parametric diagram of Successful interception rate

In order to import the model into Matlab,we set the initial value or range of values for system attributes involved in the model as follows:

Table 1 Parameter initial value setting

\begin{tabular}{lll}
\hline Equipment & Equipment performance & \multicolumn{1}{c}{ initial value } \\
\hline Early warning radar & the target echo power: Snr & $45 \mathrm{w}$ \\
& minimal signal-to-noise ratio reliability: $\mathrm{S}$ & $4.75 \mathrm{db}$ \\
Energy Test Equipment & Boltzmann constant: B & $1.38^{*} 10^{-23} \mathrm{~J} / \mathrm{k}$ \\
& reference noise figure: $\mathrm{B}_{\mathrm{nr}}$ & $2 \mathrm{k}$ \\
& noise figure: $\mathrm{N}_{\mathrm{p}}$ & 5 \\
& receiver bandwidth: $\mathrm{R}_{\mathrm{b}}$ & $1 \mathrm{GHz}$ \\
Defense missile & missile killing radius: $\mathrm{L}$ & $3 \mathrm{~m}$ \\
& guidance accuracy: $\mathrm{G}$ & $3.5 \mathrm{~m}$ \\
\hline
\end{tabular}

We import the above mentioned parametric models and the initial values in table 1 into Matlab program, the successful probability of the missile to intercept the incoming missile is 0.32874 .

\section{Result analysis and Summary}

From the calculation results can be seen, the successful probability of the missile to intercept the incoming missile is very small. It shows that it is very difficult to intercept the enemy's incoming missile successfully. In order to improve the success rate of interception we should do more in-depth research.

Next, we optimize the parameters. As shown in Fig.5, set the range of the guidance accuracy, the other parameters are constant, and analyzed the influence of guidance accuracy on the interception success rate. As can be seen from Fig.5, with the reduction of the guidance accuracy (the higher value of the guidance accuracy, the lower the accuracy of guidance), the interception success rate of intercepting the enemy's incoming missile quickly dropped. This shows that the contribution of accuracy of guidance which radar and interceptor missile make to the interception success rate is enormous. In order to improve the interception success rate of the antimissile system, it is necessary to strengthen the research of the guidance radar, and improve the precision of the guidance precision. In addition, when we design the system structure of the anti missile system, according to the corresponding relationship between the guidance accuracy and the success rate in Fig.5, the guidance 
accuracy can be determined by the interception success rate needs. This feedback mechanism can effectively solve the problems in the design of the system structure.
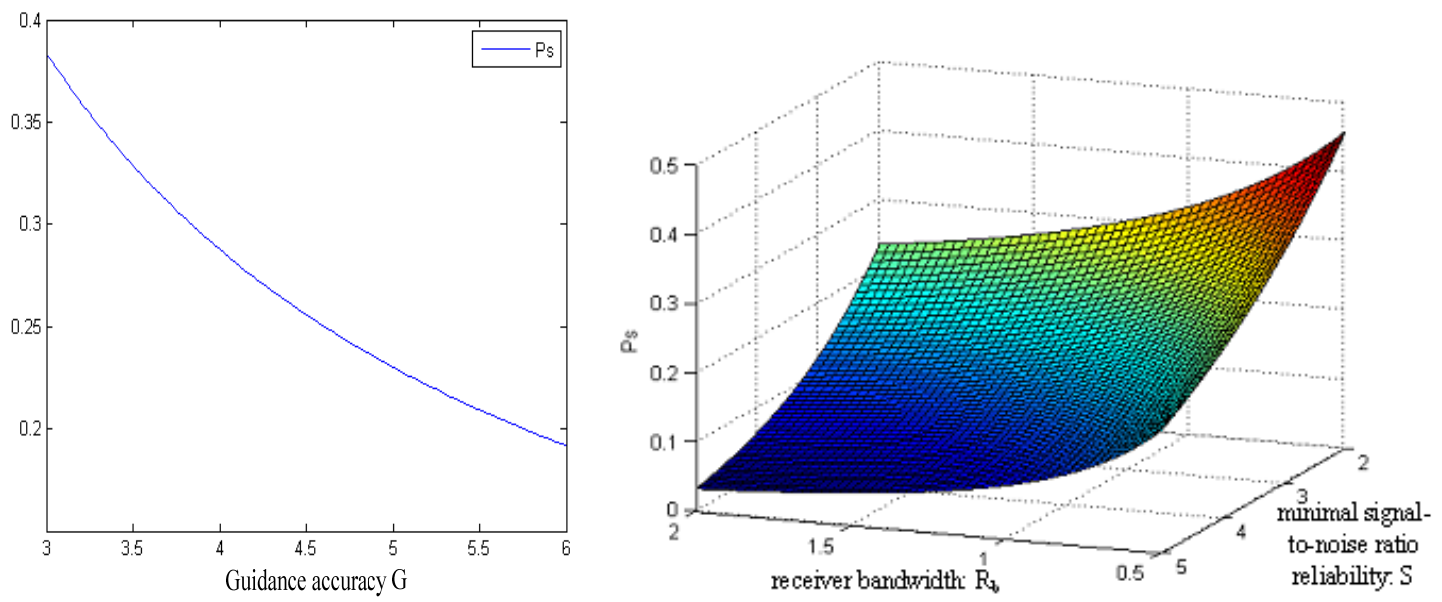

Fig.5 The influence of guidance accuracy G on Ps

Fig.6 The influence of $\mathrm{Rb}$ and $\mathrm{S}$ on $\mathrm{Ps}$

Fig. 6 shows the influence of receiver bandwidth value and the minimal signal-to-noise ratio reliability value on the interception success rate of the anti missile system. As can be seen from Fig.6, the interception success rate is increasing with the decrease of receiver bandwidth value and the minimal signal-to-noise ratio reliability value. In order to improve the interception success rate of the antimissile system, it is necessary to improve equipment performance acomplish the goal that minimize the receiver bandwidth and the minimal signal-to-noise ratio reliability.

\section{References}

[1] Han Zhaochao, Huang Shucai, Overview of evaluation method for anti-tactical ballistic missile operation. 26 (2010) 1-4.

[2] Shen Maoxing, Guo Gang, Shang Chang-an, Study of system dynamics model of anti-TBM operation of ballistic missile defense (BMD) system, Vol.14 No.3 Jun.2013,33-37

[3] Liu Jinrong, Luo Lun, Shen Yanli, Research on missile defense system architecture based on activity methodology, Vol.37 No.6 Jun, 2012, 59-62. 